\title{
Hypotension associated with ingestion of cannabinoids in two children with cancer
}

\section{Amanda M. Li MD MSc, S. Rod Rassekh MD MHSc}

Competing interests: None

declared.

This article has been peer reviewed.

The authors have obtained patient consent.

Correspondence to:

Rod Rassekh,

rrassekh@cw.bc.ca

CMAJ 2016. DOI:10.1503 /cmaj.150847

\section{Case 1}

A two-year-old boy with an ependymoma was admitted for administration of chemotherapy (cyclophosphamide, methotrexate, etoposide and cisplatin). Previous cycles of the same chemotherapy had been well tolerated, with no hypotension. Independent of medical advice, the patient's parents had been giving him $\Delta^{9}$-tetrahydrocannabinol and cannabidiol (THC-CBD) oil drops orally. Five days after his last chemotherapy dose, the patient was noted to be lethargic through the day, with downward-trending blood pressure readings.

The patient's blood pressure dropped to $50 / 30 \mathrm{~mm} \mathrm{Hg}$ during deep sleep, and his skin appeared mottled, with delayed capillary refill. His heart rate was not substantially elevated, at 107 beats/min; his temperature, respiration rate and oxygen saturation levels were normal. Upon being roused, the child was upset but responsive, with normal findings on neurologic and cardiorespiratory examination. He had maintained good urine output and did not have clinical signs suggestive of dehydration.

The patient's mother then reported that the family had independently increased the number of THC-CBD oil drops over the preceding few days from one drop three times daily to three drops three times daily. The last dose had been given 10-15 hours before the nadir in blood pressure.

The patient was given a rapid $20-\mathrm{mL} / \mathrm{kg}$ bolus of normal saline and maintenance fluid support, which corrected the hypotension. Empiric antibiotic therapy was started, although blood culture results were subsequently negative. The par-

\section{KEY POINTS}

- There is an increased use of cannabinoids offered by parents to their children during treatment of cancer.

- Cannabinoids have an effect on the cardiovascular system and substantial hypotension can result from their administration.

- There is currently no standardized dosing information for various cannabinoid formulations, and further research into their safety and efficacy is urgently needed. ents were insistent on continuing the THC-CBD oil but agreed to resume the lower dose. The patient's blood pressure remained normal, even during subsequent periods of fever and sepsis.

\section{Case 2}

A four-year-old girl at very high risk of acute lymphoblastic leukemia was admitted for a bone marrow transplant. By day 30, she had full engraftment of transplanted marrow. During this period, she received treatment for suspected fungal lesions of her spleen but did not have fungemia. Despite frequent episodes of intermittent fever, the patient was never hypotensive.

On day 30, the patient's mother administered a few drops of THC-CBD oil of unknown concentration to see if it would help alleviate symptoms of discomfort and pain. Nine hours later, while the patient was asleep, her diastolic blood pressure dropped from a baseline of $47 \mathrm{~mm} \mathrm{Hg}$ to $33 \mathrm{~mm} \mathrm{Hg}$, and her systolic pressure dropped from $99 \mathrm{~mm} \mathrm{Hg}$ to $80 \mathrm{~mm} \mathrm{Hg}$. She was febrile at $38.6^{\circ} \mathrm{C}$ and had a pulse of 120 beats/min. Over the preceding two days, the patient had been persistently febrile with normal blood pressure readings. She had been given empiric antibiotic and antifungal therapy, but blood culture results remained negative.

The patient was tired but alert and had a Glasgow Coma Scale score of 15. Her distal pulses were palpable but not bounding, and she did not appear clinically dehydrated.

The patient was given a rapid $20-\mathrm{mL} / \mathrm{kg}$ bolus of normal saline and extra maintenance intravenous fluid, and her blood pressure returned to normal. No further THC-CBD oil drops were administered, and she had no further episodes of hypotension.

\section{Discussion}

Patients with chronic or life-threatening illnesses have been using medical marijuana for symptom management and for possible anticancer activity. ${ }^{1,2}$ Cannabis has gained societal endorsement, with 
legislation allowing for its use in a medical context, and commercial clinics and dispensaries available to the public. The medical community is becoming more accepting of medical marijuana use in outpatient and inpatient settings. ${ }^{3}$ However, physicians are often caught managing the medical, legal and psychosocial aspects of cannabis without the benefit of reliable safety data and dosing parameters.

More than 60 different cannabinoids have been isolated from cannabis, the most prominent of which include THC and CBD. Cannabinoids act to varying degrees on the $\mathrm{CB} 1$ and $\mathrm{CB} 2$ receptors found in the central and peripheral nervous systems. Native cannabis and cannabinoid derivatives may have effects on symptoms such as nausea, cachexia-anorexia, neurologic and cancer-related pain, chemosensory alterations and anxiety. ${ }^{1,2,4}$ Yet there is surprisingly little scientific information to guide cannabis use in medical practice.

The cannabinoid system has been shown to modulate cardiovascular physiology in animal models. In rats, intravenous administration of anandamide, an endogenous cannabinoid primarily acting on the $\mathrm{CB} 1$ receptor, has been found to have a triphasic effect on blood pressure, characterized by a transient drop followed by a brief rise and then a sustained period of hypotension. ${ }^{5,6}$ The human cardiovascular system appears to be affected by THC. A placebo-controlled trial showed that, among people who used cannabis regularly, systolic blood pressure dropped with an oral THC dose of $30 \mathrm{mg}$ but increased at doses greater than $75 \mathrm{mg}^{7}$ A concomitant dosedependent elevation in heart rate was noted. In a randomized trial involving adults with intractable cancer-related pain, hypotension was seen in 3 of 60 participants given a commercially available spray of THC-CBD extract $(2.7 \mathrm{mg}$ THC, $2.5 \mathrm{mg} \mathrm{CBD})$ but in none of the participants in the THC-only and placebo groups. ${ }^{1}$

The effects of cannabinoids in the pediatric population are not well described. In young children, the overarching symptom of cannabis toxicity appears to be somnolence or neurologic depression. Six cases of accidental marijuana ingestion by children seen in a children's hospital resulted in coma, all reversible with supportive care. ${ }^{8}$ Of the six children, one 17-month-old child had documented hypotension. Other case reports have also described coma associated with cannabis toxicity in young children, in the absence of reported blood pressure changes. ${ }^{9}$ A systematic review of the effects of cannabinoids for the control of chemotherapy-induced nausea showed a relative risk for hypotension of 2.23 (95\% confidence interval $1.75-2.83$ ) in 30 randomized trials involving adults and children given cannabinoids (nabilone, dronabinol or levonantradol). ${ }^{4}$
In the cases we have described, causation cannot be assumed based on our observations, because both children were undergoing treatment for serious illness, and the illness itself, comorbidities and other medication could have contributed to the hypotension. Using the Naranjo Adverse Drug Reaction Probability Scale, we ranked the reaction in our two cases as "possible." 10 However, several measures of the scale, such as response to placebo and drug level measurement, were unknown in our patients and therefore could not contribute to the score. Another complicating variable is the unknown concentration and composition of the THC substance administered by the children's parents. At present, these compounds are unregulated, often extracted and processed in facilities without consistent oversight to ensure product consistency. Non-THC ingredients may have contaminated the products, causing the adverse events.

We have reported these cases to the Canada Vigilance Program, which offers consumers, health professionals and drug producers an opportunity to report adverse effects of both regulated and unregulated health products (www.hc-sc.gc. ca/dhp-mps/medeff/databasdon/index-eng.php).

\section{References}

1. Johnson JR, Burnell-Nugent M, Lossignol D, et al. Multicenter, double-blind, randomized, placebo-controlled, parallelgroup study of the efficacy, safety, and tolerability of THC:CBD extract and THC extract in patients with intractable cancer-related pain. J Pain Symptom Manage 2010;39:167-79.

2. Cannabis-In-Cachexia-Study-Group, Strasser F, Luftner D, Possinger K, et al. Comparison of orally administered cannabis extract and delta-9-tetrahydrocannabinol in treating patients with cancer-related anorexia-cachexia syndrome: a multicenter, phase III, randomized, double-blind, placebo-controlled clinical trial from the Cannabis-In-Cachexia-Study-Group. J Clin Oncol 2006; 24:3394-400.

3. Dyer O. Quebec hospitals allow inpatient use of weed. CMAJ 2014;186:E438.

4. Tramèr MR, Carroll D, Campbell FA, et al. Cannabinoids for control of chemotherapy induced nausea and vomiting: quantitative systematic review. BMJ 2001;323:16-21.

5. Varga K, Lake K, Martin BR, et al. Novel antagonist implicates the CB1 cannabinoid receptor in the hypotensive action of anandamide. Eur J Pharmacol 1995;278:279-83.

6. Lake KD, Martin BR, Kunos G, et al. Cardiovascular effects of anandamide in anesthetized and conscious normotensive and hypertensive rats. Hypertension 1997;29:1204-10.

7. Lile JA, Kelly TH, Charnigo RJ, et al. Pharmacokinetic and pharmacodynamic profile of supratherapeutic oral doses of Delta(9)THC in cannabis users. J Clin Pharmacol 2013;53:680-90.

8. Macnab A, Anderson E, Susak L. Ingestion of cannabis: a cause of coma in children. Pediatr Emerg Care 1989;5:238-9.

9. Wang GS, Narang SK, Wells K, et al. A case series of marijuana exposures in pediatric patients less than 5 years of age. Child Abuse Negl 2011;35:563-5.

10. Naranjo CA, Busto U, Sellers EM, et al. A method for estimating the probability of adverse drug reactions. Clin Pharmacol Ther 1981;30:239-45.

Affiliations: Division of Oncology, Hematology and Bone Marrow Transplant (Li, Rassekh), BC Children's Hospital; Department of Pediatrics (Rassekh), University of British Columbia, Vancouver, BC

Contributors: Both authors contributed equally to the conception and design of the article, wrote and revised it critically for important intellectual content, approved the final version to be published and agreed to act as guarantors of the work. 